\title{
Etiology of Childhood Community Acquired Pneumonia and Its Implications for Vaccination
}

Cristiana M.C. Nascimento-Carvalho
Department of Pediatrics, Faculty of Medicine, Federal University of Bahia, Salvador, Bahia, Brazil

Pneumonia is an important cause of morbidity and mortality among children throughout the world. Vaccines are available for some organisms, but they are underutilized and/or still in development. To evaluate the potential impact of vaccines, we review studies in which the etiology of childhood community-acquired pneumonia was recorded. In North America and Europe (9 studies), the etiology of pneumonia was established in $62 \%$ of studied children (range $43 \%$ $\mathbf{8 8 \%}$ ) by use of noninvasive specific methods for microbiologic diagnosis. The most often identified agents were $S$. pneumoniae (22\%), respiratory syncytial virus (RSV) (20\%), Haemophilus influenzae (7\%), and Mycoplasma pneumoniae (15\%). In Africa and South America (8 studies), bacteria were recovered from 56\% (range 32\%-68\%) of severely ill children studied by lung aspirate. The most often isolated bacteria were Streptococcus pneumoniae (33\%) and Haemophilus influenzae (21\%). A high percentage of $\boldsymbol{H}$. influenzae strains were not serotype $b$. Throughout the world, children requiring hospitalization were most likely to have infection caused by pneumococcus $H$. influenzae or RSV. Out patients also had Mycoplasma pneumoniae. Countries in Africa and Asia recorded 2 to 10 times more children with pneumonia ( 7 to $40 / 100$ annually) than in the USA. Widespread use of pneumococcal and $\boldsymbol{H}$. influenzae type b conjugate vaccines could reduce the frequency of childhood pneumonia by one-third. Further reduction will require development of non-type $b H$. influenzae, RSV and $M$. pneumoniae vaccines. This could result in a $>50 \%$ reduction of pneumonia in children. This goal should be sought and achieved as soon as possible.

Key words: community-acquired pneumonia, children, etiology, vaccine, S. pneumoniae, respiratory syncytial virus.

Acute lower respiratory tract infections (ALRTI) are a common cause of morbidity among children [1]. Among these infections, pneumonia is the most serious illness with an incidence of 36 to 40 episodes $/ 1,000$ children/year in those $<5$ years of age, and 11 to 16 episodes in children 5 to 14 years of age in the USA

Received on 12 February 2001; revised 13 March 2001.

Address for correspondence: Dr. Cristiana NascimentoCarvalho, MD, Department of Pediatrics, Faculty of Medicine, Federal University of Bahia, Rua Prof. Aristides Novis $\mathrm{N}^{\mathrm{o}}$ 105/1201B - Salvador, Bahia, Brazil - Zip Code: 40210-730. Email: otaviocr@svn.com.br

The Brazilian Journal of Infectious Diseases 2001;5(2):87-97 (C) 2001 by The Brazilian Journal of Infectious Diseases and Contexto Publishing. All rights reserved.

$1413-8670$ and in Finland [2, 3]. In poor areas of the world, the incidence of pneumonia is up to 10 times higher than that recorded above [4]. This data is summarized in Table 1 in which incidence data from the USA is compared to that of 6 countries in Africa and Asia. In these areas, pneumonia is one of the leading causes of hospitalization and death [5-7]. At present, the epidemiologic pattern of pneumonia is being altered by changes in patient characteristics, such as increased immunosuppression, and by changes in medical practice [8]. Many bacteria and viruses can cause pneumonia and many of them can be detected by methods available only in research laboratories [9]. Control of pneumonia depends upon an understanding of the relative importance of etiologic agents for recommendations regarding treatment and for the development of vaccines [10]. 
In this report, we reviewed the literature to identify studies of childhood community-acquired pneumonia in which the etiology was assessed by either lung aspiration or noninvasive, but specific, methods to study viruses and bacteria. This information has been summarized in order to assess the potential for an impact by vaccine prevention.

\section{Defining the etiology}

The difficulties in establishing a firm etiology for patients with pneumonia are well known. Culture of sputum is of uncertain value and samples of sputum are difficult to obtain from children [11]; the degree of concordance between pharyngeal secretion cultures and lung aspirate cultures was poor [12]; blood cultures yield a positive result in $1 \%$ to $27 \%$ of patients $[9,10$, 13-15], and its isolation rate is highly dependent on when during health care it is done [16], and on which patients are recruited. It was shown that identification of nonbacteremic pneumococcal infections in children is difficult and confusing because different assays give different results [9, 17-19]. For optimal yield, several techniques should be used even for the detection of 1 microbe [9]. As yet, there are no sensitive and simple tests for the detection of the etiologic agents in biological fluids from patients with pneumonia. Consequently, there is a great shortage of data necessary for implementing potentially effective intervention measures [20]. Ideally, the determination of etiology should be based on isolation of the organism from infected lung tissue, as any growth from a needle aspirate of the lungs appears to be significant [21].

The information gained from 8 studies in which lung aspiration for culture was done are shown in Table 2. Since only culture was used, there is no information on virologic causes of childhood pneumonia. Criteria to be included was no history of recent antibiotic use in the studies from Africa, South America and Asia. Bacteria were recovered from 273 of 490 (56\%) critically ill children studied [11, 12, 21-26]. The bacteria most often isolated were Streptococcus pneumoniae and Haemophilus influenzae, including both organisms several times. $19 \%$ of the $H$. influenzae strains typed were type $b$ and the majority were other serotypes (25\%) or nonserotypable types (NST) (56\%) $[11,25]$. The sensitivity of biopsy or needle aspiration of lung tissue was less than $100 \%$ [27]. Aspiration of lung tissue is a very invasive procedure, and was restricted to critically ill children. It is rarely done at the present time. However, in many countries of the world, this approach has yielded the only firm data on the frequency of various etiologic causes of pneumonia.

Noninvasive techniques can be used to seek evidence of viral and bacterial pulmonary infection, making it possible to study children with mild disease who could not ethically be studied for bacterial infection using lung aspiration [28]. Table 3 shows the summary of specific noninvasive methods for microbiologic diagnosis of the etiology of pneumonia, performed in several studies (see results in Tables 4 and 5) which results are shown in Tables 4 and $5[9,10,13,14,18$, 29-32]. In addition to the pathogens listed in Tables 4 and 5, Staphylococcus aureus, Streptococcus pyogenes, Chlamydia pneumoniae, Chlamydia trachomatis, Legionella pneumophila, herpes virus, enterovirus, rotavirus, coronavirus, Epstein-Barr virus and varicella-zoster were also identified in several of those studies. Those studies were conducted in the USA, Finland, Spain, and Sweden. The etiology was established in $62 \%$ of the children studied (including both out patients and hospitalized children) and $S$. pneumoniae was identified as an important cause of pneumonia in all age groups. Respiratory syncytial virus (RSV) and Mycoplasma pneumoniae were the other most frequently identified agents. The classic view is that viruses probably do have a role in the initiation of bacterial invasion in pneumonia [33]. In this review, it is noted that respiratory viruses were present in $36 \%$ of the patients and mixed viral-bacterial infection was detected in $13 \%$ of the pneumonia cases.

RSV has long been recognized as the major viral pathogen of the lower respiratory tract of infants [34]. In Pakistan, RSV was identified in $33 \%$ of children with ALRTI.[35] In 148 Filipino children with ALRTI, evidence for viral infection was found for RSV (44.6\%), adenovirus (18.2\%), parainfluenza $3(11.5 \%)$, influenza A $(7.4 \%)$, influenza B $(6.8 \%)$, parainfluenza $1(6.1 \%)$ and parainfluenza $2(5.4 \%)$ [36]. In South Africa, acute 
Table 1. Examples of annual incidence of pneumonia in USA Compared to 5 Countries in Africa/Asia (Pechere J.C., 1995)

\begin{tabular}{llc}
\hline Location & Age (yr) & $\begin{array}{c}\text { Annual incidence per 100 } \\
\text { children }\end{array}$ \\
\hline United States of America & $<5$ & 3.6 \\
$\quad$ Chapel Hill, NC & $<5$ & 3.0 \\
$\quad$ Seattle, WA & $<5$ & 18.0 \\
Africa & & \\
$\quad$ Maragna, Kenya & $<5$ & 7.0 \\
Asia & $<5$ & 13.0 \\
$\quad$ Bangkok, Thailand & $<5$ & 30.0 \\
Gadchiorli, India & $<1 *$ & 40.0 \\
Gilgit, Pakistan & $<1 \dagger$ & 30.0 \\
Haryana, India & $<1$ & 25.6 \\
$\quad$ Papua New Guinea & & \\
\hline
\end{tabular}

*Low birth weight; $\uparrow$ Normal birth weight.

respiratory tract infections caused approximately $8 \%$ of all deaths in the under-5 age group; the published hospital-based incidence of RSV infection varied from $3 \%$ to $18 \%$, and mortality rates were between $12 \%$ and $43 \%$ [37]. Currently available information from laboratory records and published South African literature is not sufficient to assess the impact of RSV infection [37]. In Hong Kong, the estimated annual incidence of RSV infection requiring hospitalization was $2.5 / 1000$ children $<5$ years old with a mortality of $0.15 \%$ among hospitalized cases and a mean hospital stay of 5 days. RSV vaccine is considered a priority by several experts [38]. RSV frequency has ranged from $15.8 \%-28 \%$ in several other studies conducted in Argentina, Ethiopia, Japan and Mexico, studying children with ALRTI [39-42]. Despite its important frequency, distinguishing RSV from other ALRTIs is difficult because of the similarities in clinical presentation [43]; RSV has also been associated with recurrent episodes of wheezing in children [44].

M. pneumoniae has been found in $27 \%$ to $38 \%$ of children with pneumonia studied in India, Japan and Poland [45-47]. Two other prospective studies reported evidence of infection with $M$. pneumoniae in $27 \%$ to $29.5 \%$, and with Chlamydia pneumoniae in $15 \%$ to $28 \%$ of children with community-acquired pneumonia in the USA [48, 49].

The data presented here support the view that the most serious forms of pneumonia in children of all ages are pneumococcus, $H$. influnenzae and respiratory syncitial virus. These are the agents against which vaccines are most needed, and, indeed, are most likely soon to be available. These are the agents that are also most likely to be the cause of pneumonia in children under 5 years of age. Mycoplasma pneumoniae and chlamydial pneumonia occur in older children and are less likely to require hospitalization. Viral pneumonia occurs most commonly in the very young, under age 5 years but, other than RSV, they are not as common a cause of hospitalization as are bacterial pneumonias. From this data, it can be concluded that the most important causes of morbidity and mortality in childhood pneumonia can be prevented by vaccines that are either now, or soon to be available. 
Table 2. Bacteriologic findings in studies from Africa, Asia and South America evaluated using lung aspirates

\begin{tabular}{|c|c|c|c|c|c|c|c|}
\hline \multirow[t]{2}{*}{ Study } & \multirow[t]{2}{*}{ Country } & \multirow[t]{2}{*}{ Age (years) } & \multicolumn{5}{|c|}{$\mathrm{N}^{0}$ of positive bacteria isolated } \\
\hline & & & Culture/total & S. pneumoniae & H. influenzae & S. aureus & Others \\
\hline Schuster et al., 1966 & Chile & $\leq 10$ & $67 / 125(54 \%)$ & $26 / 125(21 \%)$ & $19 / 125(15 \%)$ & $15 / 125(12 \%)$ & $13 / 125(10 \%)$ \\
\hline Rozov et al., 1974 & Brazil & $\leq 7$ & $20 / 37(54 \%)$ & $15 / 37(41 \%)$ & $3 / 37(8 \%)$ & $1 / 37(3 \%)$ & $1 / 37(3 \%)$ \\
\hline Silverman et al., 1977 & Nigeria & $\leq 8$ & $54 / 88(61 \%)$ & $31 / 88(35 \%)$ & $9 / 88(10 \%)$ & $8 / 88(9 \%)$ & $20 / 88(23 \%)$ \\
\hline Ferreira et al., 1978 & Brazil & $\leq 4$ & $34 / 60(57 \%)$ & $25 / 60(42 \%)$ & $13 / 60(22 \%)$ & 0 & 0 \\
\hline Riley et al., 1983 & Papua & $\leq 9$ & $8 / 18(44 \%)$ & $7 / 18(39 \%)$ & $1 / 18(6 \%)$ & 0 & 0 \\
\hline & New Guinea & & & & & & \\
\hline Shann et al., 1984 & Papua & $\leq 5$ & $48 / 71(68 \%)$ & $27 / 71(38 \%)$ & $41 / 71(58 \%)$ & $1 / 71(1 \%)$ & $23 / 71(32 \%)$ \\
\hline & New Guinea & & & & & & \\
\hline Wall et al., 1986 & The Gambia & $\leq 9$ & $29 / 51(57 \%)$ & $26 / 51(51 \%)$ & $12 / 51(24 \%)$ & $1 / 51(2 \%)$ & $2 / 51(4 \%)$ \\
\hline Ikeogu et al., 1988 & Zimbabwe & $\leq 11$ & $13 / 40(32 \%)$ & $7 / 40(18 \%)$ & $3 / 40(8 \%)$ & $4 / 40(10 \%)$ & $1 / 40(2 \%)$ \\
\hline Total & & & $273 / 490(56 \%)$ & $164 / 490(33 \%)$ & $101 / 490(21 \%)$ & $30 / 490(6 \%)$ & $60 / 490(12 \%)$ \\
\hline Microbe Cause/ & & & & & & & \\
\hline Detected & & & & $164 / 273(60 \%)$ & 101/273 (37\%) & $30 / 273(11 \%)$ & $60 / 273(22 \%)$ \\
\hline
\end{tabular}


Table 3. Summary of noninvasive specific methods for microbiologic diagnosis of pneumonia*

\begin{tabular}{|c|c|c|}
\hline Agents and assays & Assay & Diagnostic finding \\
\hline \multicolumn{3}{|l|}{$\overline{\text { Streptococcus pneumoniae }}$} \\
\hline \multicolumn{3}{|l|}{ Antibodies } \\
\hline To capsular polysaccharides & EIA & 3-fold rise \\
\hline To C-polysaccharide & EIA & 3 -fold rise \\
\hline Topneumolysin & EIA & 2-fold rise \\
\hline \multicolumn{3}{|l|}{ Immune complexes specific } \\
\hline To capsular polysaccharides & EIA & $\mathrm{GM}+\mathrm{SD}$ \\
\hline To C-polysaccharide & EIA & $\mathrm{GM}+\mathrm{SD}$ \\
\hline Topneumolysin & EIA & $\mathrm{GM}+\mathrm{SD}$ \\
\hline Antigens & CIE, LA, CoA & \\
\hline \multicolumn{3}{|l|}{ H. influenzae } \\
\hline Antibodies(noncapsulated $H . i$.) & EIA & 3-fold rise \\
\hline Antigens & CIE, LA & \\
\hline \multicolumn{3}{|l|}{ Moraxella catarrahalis } \\
\hline Antibodies & EIA & 3-fold rise \\
\hline \multicolumn{3}{|l|}{ Chlamydiae } \\
\hline \multicolumn{3}{|l|}{ Antibodies } \\
\hline To re-lipopolysaccharide & EIA & 3-fold rise \\
\hline To common group antigen & $\mathrm{CF}$ & 3-fold rise \\
\hline Chlamydia pneumoniae & MIF & 4 -fold rise or $\operatorname{IgM}>1 / 16$ \\
\hline Chlamydia psittaci & Culture, PCR & \\
\hline Chlamydia trachomatis & MIF & 4 -fold rise or $\operatorname{IgM}>1 / 16$ \\
\hline \multirow[t]{3}{*}{ Mycoplasma pneumoniae } & CF, EIA & 3 -fold rise \\
\hline & IgM-EIA & \\
\hline & Culture, PCR & \\
\hline Legionella & IFA & \\
\hline Respiratory viruses & Culture & \\
\hline \multirow[t]{2}{*}{ Antibodies } & $\mathrm{CF}$ & 4-fold rise \\
\hline & $\begin{array}{l}\text { EIA*, MIF, DFA, } \\
\text { MNA }\end{array}$ & *3-fold rise \\
\hline \multirow[t]{3}{*}{ Antigens } & Reverse-transcriptase & \\
\hline & PCR, FIA & \\
\hline & Solid phase EIA & \\
\hline
\end{tabular}

*Modified from Heiskanen-Kosma et al. [31]; EIA: enzyme immunoassay; GM+SD: geometric mean + 2SD; CIE: counterimmunoelectrophoresis; LA: latex agglutination; CoA: coagglutination; CF: complement-fixation; MIF: microimmunofluorescence; MNA: microtiter neutralization assay; IFA: indirect immunofluorescent antibody H.i.: H. influenzae; DFA: viral direct fluorescent antibody; FIA:time-resolved fluoroimmunoasay with monoclonal antibodies. 
Table 4. Etiology of childhood community-acquired pneumonia in Europe using non-invasive diagnostic techniques - hospitalized patients

\begin{tabular}{|c|c|c|c|c|c|c|c|c|c|c|c|c|c|c|c|c|}
\hline Study & $\mathbf{N}$ & Country & Age & $\begin{array}{l}\text { Etiology } \\
\text { detected } \\
\text { (total) }\end{array}$ & $\begin{array}{c}\text { Total } \\
\text { bacteria }\end{array}$ & $\begin{array}{c}S . \\
\text { pneumo- } \\
\text { niae }\end{array}$ & $\begin{array}{c}\text { H. } \\
\text { influ- } \\
\text { enzae }\end{array}$ & $\begin{array}{c}M . \\
\text { pneumo- } \\
\text { niae }\end{array}$ & $\begin{array}{c}M . \\
\text { catar } \\
\text { rhalis }\end{array}$ & $\begin{array}{c}\text { Total } \\
\text { viruses }\end{array}$ & $\begin{array}{c}\text { Respir- } \\
\text { atory } \\
\text { syncytial } \\
\text { virus }\end{array}$ & $\begin{array}{c}\text { Adeno- } \\
\text { virus }\end{array}$ & $\begin{array}{c}\text { Rhino- } \\
\text { virus }\end{array}$ & $\begin{array}{l}\text { Influ- } \\
\text { enza A } \\
\text { or B }\end{array}$ & $\begin{array}{c}\text { Para- } \\
\text { influ- } \\
\text { enza } \\
1,2,3\end{array}$ & $\begin{array}{c}\text { Mixed } \\
\text { viral } \\
\text { bacterial }\end{array}$ \\
\hline $\begin{array}{l}\text { Claesson } \\
\text { et al., } \\
1989\end{array}$ & 167 & Sweden & $\begin{array}{c}\text { Median } \\
1 \mathrm{yr}\end{array}$ & $\begin{array}{c}71 / 167 \\
(43 \%)\end{array}$ & $\begin{array}{l}32 / 167 \\
(19 \%)\end{array}$ & $\begin{array}{l}27 / 167 \\
(16 \%)^{*}\end{array}$ & $\begin{array}{l}1 / 167 \\
\text { tybe b } \\
(0.6 \%)\end{array}$ & $\begin{array}{c}6 / 167 \\
(4 \%)\end{array}$ & NS & $\begin{array}{c}45 / 167 \\
(27 \%)\end{array}$ & $\begin{array}{l}33 / 167 \\
(20 \%)\end{array}$ & $\begin{array}{c}11 / 167 \\
(7 \%)\end{array}$ & NS & $\begin{array}{l}3 / 167 \\
(2 \%)\end{array}$ & $\begin{array}{l}5 / 167 \\
(3 \%)\end{array}$ & $\begin{array}{c}6 / 167 \\
(4 \%)\end{array}$ \\
\hline $\begin{array}{l}\text { Nohynek } \\
\text { et al., } \\
1991\end{array}$ & 121 & Finland & $\begin{array}{c}\text { Median } \\
1.8 \mathrm{yr}\end{array}$ & $\begin{array}{c}84 / 121 \\
(69 \%)\end{array}$ & $\begin{array}{c}54 / 121 \\
(45 \%)\end{array}$ & $\begin{array}{l}19 / 121 \\
(16 \%)^{*}\end{array}$ & $\begin{array}{c}21 / 121 \\
(17 \%)\end{array}$ & $\begin{array}{c}11 / 121 \\
(9 \%)\end{array}$ & $\begin{array}{l}9 / 121 \\
(7 \%)\end{array}$ & $\begin{array}{c}54 / 121 \\
(45 \%)\end{array}$ & $\begin{array}{l}34 / 121 \\
(28 \%)\end{array}$ & $\begin{array}{l}16 / 121 \\
(13 \%)\end{array}$ & NS & $\begin{array}{c}4 / 121 \\
(3 \%)\end{array}$ & $\begin{array}{c}8 / 121 \\
(7 \%)\end{array}$ & $\begin{array}{c}24 / 121 \\
(20 \%)\end{array}$ \\
\hline $\begin{array}{l}\text { Ruuskanen } \\
\text { et al., } \\
1992\end{array}$ & 50 & Finland & $\begin{array}{l}\text { Mean } \\
4.4 \mathrm{yr}\end{array}$ & $\begin{array}{l}44 / 50 \\
(88 \%)\end{array}$ & $\begin{array}{l}31 / 50 \\
(62 \%)\end{array}$ & $\begin{array}{l}19 / 50 \\
(38 \%)\end{array}$ & $\begin{array}{c}6 / 50 \\
(12 \%)\end{array}$ & $\begin{array}{l}10 / 50 \\
(20 \%)\end{array}$ & $\begin{array}{c}5 / 50 \\
(10 \%)\end{array}$ & $\begin{array}{l}30 / 50 \\
(60 \%)\end{array}$ & $\begin{array}{l}15 / 50 \\
(30 \%)\end{array}$ & $\begin{array}{c}5 / 50 \\
(10 \%)\end{array}$ & $\begin{array}{c}5 / 50 \\
(10 \%)\end{array}$ & $\begin{array}{l}1 / 50 \\
(2 \%)\end{array}$ & $\begin{array}{l}4 / 50 \\
(8 \%)\end{array}$ & $\begin{array}{l}17 / 50 \\
(34 \%)\end{array}$ \\
\hline $\begin{array}{l}\text { Juvén } \\
\text { et al., } \\
2000\end{array}$ & 254 & Finland & $\begin{array}{l}\text { Mean } \\
3.8 \mathrm{yr}\end{array}$ & $\begin{array}{c}215 / 254 \\
(85 \%)\end{array}$ & $\begin{array}{c}134 / 254 \\
(53 \%)\end{array}$ & $\begin{array}{c}93 / 254 \\
(37 \%)\end{array}$ & $\begin{array}{c}22 / 254 \\
(9 \%)\end{array}$ & $\begin{array}{c}17 / 254 \\
(7 \%)\end{array}$ & $\begin{array}{c}10 / 254 \\
(4 \%)\end{array}$ & $\begin{array}{c}158 / 254 \\
(62 \%)\end{array}$ & $\begin{array}{c}73 / 254 \\
(29 \%)\end{array}$ & $\begin{array}{c}19 / 254 \\
(7 \%)\end{array}$ & $\begin{array}{c}58 / 238 \\
(24 \%)\end{array}$ & $\begin{array}{c}10 / 254 \\
(4 \%)\end{array}$ & $\begin{array}{c}25 / 254 \\
(10 \%)\end{array}$ & $\begin{array}{c}77 / 254 \\
(30 \%)\end{array}$ \\
\hline Total & 592 & & & $\begin{array}{c}414 / 592 \\
(70 \%)\end{array}$ & $\begin{array}{c}251 / 592 \\
(42 \%)\end{array}$ & $\begin{array}{c}158 / 592 \\
(27 \%)\end{array}$ & $\begin{array}{c}49 / 425^{* *} \\
(12 \%)\end{array}$ & $\begin{array}{c}44 / 592 \\
(7 \%)\end{array}$ & $\begin{array}{c}24 / 425 * * \\
* * \\
(6 \%)\end{array}$ & $\begin{array}{c}287 / 592 \\
(48 \%)\end{array}$ & $\begin{array}{c}155 / 592 \\
(26 \%)\end{array}$ & $\begin{array}{c}51 / 592 \\
(9 \%)\end{array}$ & $\begin{array}{c}63 / 288 \\
* * * \\
(22 \%)\end{array}$ & $\begin{array}{c}18 / 592 \\
(3 \%)\end{array}$ & $\begin{array}{c}42 / 592 \\
(7 \%)\end{array}$ & $\begin{array}{c}124 / 592 \\
(21 \%)\end{array}$ \\
\hline Microbe & $\mathrm{X}$ & & & & $251 / 414$ & $158 / 414$ & $49 / 343$ & $44 / 414$ & $24 / 343$ & $287 / 414$ & $155 / 414$ & $51 / 414$ & $63 / 259$ & $18 / 414$ & $42 / 414$ & $124 / 414$ \\
\hline $\begin{array}{l}\text { Cause/ } \\
\text { Detected }\end{array}$ & 414 & & & & $(61 \%)$ & (38\%) & $\begin{array}{c}* * \\
(14 \%)\end{array}$ & $(11 \%)$ & $\begin{array}{c}* * \\
(7 \%)\end{array}$ & $(69 \%)$ & $(37 \%)$ & $(12 \%)$ & $\begin{array}{c}* * * \\
(24 \%)\end{array}$ & $(4 \%)$ & $(10 \%)$ & $(30 \%)$ \\
\hline
\end{tabular}

NS = not studied; * low percentage in very young infants believed by the authors due to poor immune response. Organism was present in nasopharynx (25\%).

** Claesson study excluded because only type b $H$. infuenzae was tested and $M$. catarrhalis was not tested.

$* * *$ rhinovirus not tested in 2 studies. 
Table 5. Etiology of chilhood pneumonia in North America and Europe diagnosied by non-invasive techniques - primarily out-patient children

\begin{tabular}{|c|c|c|c|c|c|c|c|c|c|c|c|c|c|c|c|c|}
\hline Study & $\mathrm{N}$ & Country & Age & $\begin{array}{l}\text { Etiology } \\
\text { detected } \\
\text { (total) }\end{array}$ & $\begin{array}{c}\text { Total } \\
\text { bacteria }\end{array}$ & $\begin{array}{c}S . \\
\text { pneumo- } \\
\text { niae }\end{array}$ & $\begin{array}{c}H . \\
\text { influ- } \\
\text { enzae }\end{array}$ & $\begin{array}{c}\text { M. } \\
\text { pneumo- } \\
\text { niae }\end{array}$ & $\begin{array}{l}M . \\
\text { catar- } \\
\text { rhalis }\end{array}$ & $\begin{array}{c}\text { Total } \\
\text { viruses }\end{array}$ & $\begin{array}{l}\text { Respir- } \\
\text { atory } \\
\text { syncytial } \\
\text { virus }\end{array}$ & $\begin{array}{l}\text { Adeno- } \\
\text { virus }\end{array}$ & $\begin{array}{l}\text { Rhino- } \\
\text { virus }\end{array}$ & $\begin{array}{c}\text { Influ- } \\
\text { enza A } \\
\text { or B }\end{array}$ & $\begin{array}{l}\text { Para- } \\
\text { influ- } \\
\text { enza } \\
1,2,3\end{array}$ & $\begin{array}{l}\text { Mixed } \\
\text { viral } \\
\text { bacterial }\end{array}$ \\
\hline $\begin{array}{l}\text { Ramsey } \\
\text { et al., } \\
1986^{*}\end{array}$ & 162 & USA & $\begin{array}{c}\text { Median } \\
21 \mathrm{mo}\end{array}$ & $\begin{array}{c}77 / 162 \\
(48 \%)\end{array}$ & $\begin{array}{c}47 / 162 \\
(29 \%)\end{array}$ & $\begin{array}{c}29 / 162 \\
(18 \%)\end{array}$ & $\begin{array}{c}12 / 162 \\
(7 \%)\end{array}$ & $\begin{array}{c}13 / 162 \\
(8 \%)\end{array}$ & NS & $\begin{array}{l}50 / 162 \\
(31 \%)\end{array}$ & $\begin{array}{c}15 / 162 \\
(9 \%)\end{array}$ & $\begin{array}{l}6 / 162 \\
(4 \%)\end{array}$ & $\begin{array}{l}6 / 162 \\
(4 \%)\end{array}$ & $\begin{array}{c}10 / 162 \\
(6 \%)\end{array}$ & $\begin{array}{c}10 / 162 \\
(6 \%)\end{array}$ & $\begin{array}{c}13 / 162 \\
(8 \%)\end{array}$ \\
\hline $\begin{array}{l}\text { Turner } \\
\text { et al., } \\
1987 *\end{array}$ & 98 & USA & $\begin{array}{c}\text { Median } \\
<2 \mathrm{yr}\end{array}$ & $\begin{array}{l}47 / 98 \\
(48 \%)\end{array}$ & $\begin{array}{l}19 / 98 \\
(19 \%)\end{array}$ & $\begin{array}{l}17 / 98 \\
(17 \%)\end{array}$ & $\begin{array}{l}2 / 98 \\
(2 \%)\end{array}$ & 0 & NS & $\begin{array}{l}39 / 98 \\
(39 \%)\end{array}$ & $\begin{array}{l}27 / 98 \\
(28 \%)\end{array}$ & $\begin{array}{l}1 / 98 \\
(1 \%)\end{array}$ & $\begin{array}{l}2 / 98 \\
(2 \%)\end{array}$ & $\begin{array}{l}2 / 98 \\
(2 \%)\end{array}$ & $\begin{array}{l}5 / 98 \\
(5 \%)\end{array}$ & $\begin{array}{l}10 / 98 \\
(1 \%)\end{array}$ \\
\hline $\begin{array}{l}\text { Ausina } \\
\text { et al., } \\
1988^{*}\end{array}$ & 198 & Spain & $\begin{array}{l}\text { Median } \\
5 \mathrm{yr}\end{array}$ & $\begin{array}{c}157 / 198 \\
(79 \%)\end{array}$ & $\begin{array}{c}115 / 198 \\
(58 \%)\end{array}$ & $\begin{array}{c}29 / 198 \\
(15 \%)\end{array}$ & $\begin{array}{c}3 / 198 \\
(2 \%)\end{array}$ & $\begin{array}{l}79 / 198 \\
(40 \%)\end{array}$ & NS & $\begin{array}{l}53 / 198 \\
(27 \%)\end{array}$ & $\begin{array}{c}29 / 198 \\
(15 \%)\end{array}$ & $\begin{array}{l}5 / 198 \\
(3 \%)\end{array}$ & NS & $\begin{array}{l}8 / 198 \\
(4 \%)\end{array}$ & $\begin{array}{c}10 / 198 \\
(5 \%)\end{array}$ & NS \\
\hline $\begin{array}{l}\text { Heiska- } \\
\text { nen-Kosma } \\
\text { et al.,1998 }\end{array}$ & $\begin{array}{l}201 \\
* *\end{array}$ & $\begin{array}{c}\text { Finland } \\
5.6 \mathrm{yr}\end{array}$ & $\begin{array}{l}\text { Mean } \\
(66 \%)\end{array}$ & $\begin{array}{c}133 / 201 \\
(51 \%)\end{array}$ & $\begin{array}{c}102 / 201 \\
(28 \%)\end{array}$ & $\begin{array}{c}57 / 201 \\
(6 \%)\end{array}$ & $\begin{array}{l}12 / 201 \\
(22 \%)\end{array}$ & $\begin{array}{c}44 / 201 \\
(3 \%)\end{array}$ & $\begin{array}{l}6 / 201 \\
(25 \%)\end{array}$ & $\begin{array}{l}51 / 201 \\
(21 \%)\end{array}$ & $\begin{array}{c}43 / 201 \\
(2 \%)\end{array}$ & $4 / 201$ & NS & $\begin{array}{c}0 \\
(3 \%)\end{array}$ & $\begin{array}{l}6 / 201 \\
(10 \%)\end{array}$ & $20 / 201$ \\
\hline $\begin{array}{l}\text { Wubbel } \\
\text { et al., } \\
1999\end{array}$ & 168 & USA & $\begin{array}{l}\text { Median } \\
3 \mathrm{yr}\end{array}$ & $\begin{array}{l}73 / 168 \\
(43 \%)\end{array}$ & $\begin{array}{l}47 / 168 \\
(28 \%)\end{array}$ & $\begin{array}{l}35 / 129 \\
(27 \%)\end{array}$ & NS & $\begin{array}{c}12 / 168 \\
(7 \%)\end{array}$ & NS & $\begin{array}{l}31 / 157 \\
(20 \%)\end{array}$ & $\begin{array}{c}13 / 157 \\
(8 \%)\end{array}$ & $\begin{array}{l}3 / 157 \\
(2 \%)\end{array}$ & NS & $\begin{array}{l}5 / 157 \\
(3 \%)\end{array}$ & $\begin{array}{l}7 / 157 \\
(4 \%)\end{array}$ & $\begin{array}{l}5 / 168 \\
(3 \%)\end{array}$ \\
\hline $\begin{array}{l}\text { Claesson } \\
\text { et al., } \\
1989\end{array}$ & 169 & Sweden & $\begin{array}{c}\text { Median } \\
5 \mathrm{yr}\end{array}$ & $\begin{array}{l}89 / 169 \\
(53 \%)\end{array}$ & $\begin{array}{l}45 / 169 \\
(27 \%)\end{array}$ & $\begin{array}{c}17 / 169 \\
(10 \%)\end{array}$ & $\begin{array}{c}2 / 169 \\
\text { type b } \\
(1 \%)\end{array}$ & $\begin{array}{c}27 / 169 \\
(15 \%)\end{array}$ & NS & $\begin{array}{l}52 / 169 \\
(31 \%)\end{array}$ & $\begin{array}{l}34 / 169 \\
(20 \%)\end{array}$ & $\begin{array}{l}7 / 169 \\
(4 \%)\end{array}$ & NS & $\begin{array}{l}7 / 169 \\
(4 \%)\end{array}$ & $\begin{array}{l}6 / 169 \\
(4 \%)\end{array}$ & $\begin{array}{l}8 / 169 \\
(7 \%)\end{array}$ \\
\hline $\begin{array}{l}\text { Total } \\
\text { Microbe }\end{array}$ & $\begin{array}{c}996 \\
\mathrm{X} /\end{array}$ & & & $\begin{array}{c}576 / 996 \\
(58 \%)\end{array}$ & $\begin{array}{c}375 / 996 \\
(38 \%)\end{array}$ & $\begin{array}{c}184 / 957 \\
(19 \%)\end{array}$ & $\begin{array}{c}29 / 659 \\
(4 \%)\end{array}$ & $\begin{array}{c}175 / 898 \\
(19 \%)\end{array}$ & $\begin{array}{l}6 / 201 \\
(3 \%)\end{array}$ & $\begin{array}{c}275 / 985 \\
(28 \%)\end{array}$ & $\begin{array}{c}161 / 985 \\
(16 \%)\end{array}$ & $\begin{array}{c}26 / 985 \\
(3 \%)\end{array}$ & $\begin{array}{c}8 / 260 \\
(3 \%)\end{array}$ & $\begin{array}{c}32 / 784 \\
(4 \%)\end{array}$ & $\begin{array}{c}44 / 985 \\
(4 \%)\end{array}$ & $\begin{array}{c}56 / 798 \\
(7 \%)\end{array}$ \\
\hline $\begin{array}{l}\text { Cause/ } \\
\text { Detected }\end{array}$ & 576 & & & & $\begin{array}{c}375 / 576 \\
(65 \%)\end{array}$ & $\begin{array}{c}184 / 576 \\
(32 \%)\end{array}$ & $\begin{array}{c}29 / 414 \\
(7 \%)\end{array}$ & $\begin{array}{c}175 / 529 \\
(33 \%)\end{array}$ & $\begin{array}{c}6 / 133 \\
(5 \%)\end{array}$ & $\begin{array}{c}275 / 576 \\
(48 \%)\end{array}$ & $\begin{array}{c}161 / 576 \\
(28 \%)\end{array}$ & $\begin{array}{c}26 / 576 \\
(5 \%)\end{array}$ & $\begin{array}{l}8 / 124 \\
(6 \%)\end{array}$ & $\begin{array}{c}32 / 443 \\
(7 \%)\end{array}$ & $\begin{array}{c}44 / 576 \\
(8 \%)\end{array}$ & $\begin{array}{c}56 / 419 \\
(13 \%)\end{array}$ \\
\hline
\end{tabular}

$* 19 \%$ to $21 \%$ of patients required hospitalization. $* * 32 \%$ of patients required hospitalization. 


\section{Prevention}

The etiology of childhood community-acquired pneumonia is varied and establishing its etiology is complex. Nonetheless, from both groups of studies listed (Tables 2, 4 and 5), S. pneumoniae was the most frequently identified agent. S. pneumoniae was also found to be associated with death in children with pneumonia [28]. Pneumococcal polysaccharide vaccines have been recommended since 1985, for children older than 2 years who are at high risk of invasive disease (for example asplenic children). These vaccines have not been recommended for younger children and infants because of poor antibody response before 2 years of age [50]. In contrast, pneumococcal conjugate vaccine (Prevnar) induces protective antibody responses in $>90 \%$ of infants after 3 doses are given at 2, 4, and 6 months of age [51]. In efficacy trials, infant immunization with Prevnar decreased pneumonia by $73 \%$ [52]. The Food and Drug Administration (FDA) and the Committee on Infectious Diseases of the American Academy of Pediatrics have recently approved the use of Prevnar in children younger than 24 months in the USA [52]. However, it is clear that the efficacy of Prevnar, or any other conjugate pneumococcal vaccine, will depend on the serotype distribution of $S$. pneumoniae in the region where the vaccine is to be used [53, 54]. For example, by comparing Prevnar serotypes with the prevalence of serotypes among 360 pneumococcus strains isolated from children with invasive disease in three cities of Brazil, Prevnar would prevent $55 \%$ of pneumococcal invasive disease [55]. Since the licensure of conjugate $H$. influenzae type b (Hib) vaccines in late 1990, there has been a $>98 \%$ elimination of Hib disease in the USA [56]. Hib is a very important cause of primarily invasive diseases like meningitis and bacteremia [57].

Pneumonia can also be an invasive disease but secondarily because the initial contamination route is the respiratory airway [58]. Serotype b is more likely to invade the bloodstream from the lung than NST strains [56]. It is believed that $H$. influenzae pneumonia is usually due to serotype b because of results from blood cultures [59]. However, Ghafoor et al., reported that nonencapsulated $H$. influenzae accounted for $32 \%$ of the Haemophilus isolated from the blood of children with acute lower respiratory tract infections in Pakistan [35]. It has been shown that Hib was found in just $19 \%$ of pneumonia caused by $H$. influenzae in lung aspirate investigations [11, 25]. Because the conjugate $H$. influenzae vaccine is restricted to serotype $b$, we can expect that the wide use of the conjugate Hib vaccines would reduce about $20 \%$ to $30 \%$ of pneumonia caused by $H$. influenzae. A RSV effective vaccine is not currently available.

New approaches to the development of a vaccine are promising [60]. However, phase III efficacy trials in infants and young children are still required before final approval [61]. The current trend has been to identify subunit vaccines [62], or a live RSV intranasally administered vaccine. Both of these vaccines are immunogenic and safe in children and adults, and the latter also protects against RSV-induced airway hyperresponsiveness in the setting of allergic sensitization [63]. Recent advances in RSV vaccines have made RSV a more important topic for epidemiological research and surveillance. Basic research required before vaccine programs can be developed includes quantifying the burden of disease attributable to RSV, and defining the best surveillance methods with which to evaluate different vaccination strategies [64]. M. pneumoniae oral vaccination offers a promising route for stimulating protective immunity while minimizing undesirable recall immune events, but those studies are still restricted to animal models [65].

\section{Conclusion}

The etiology of childhood community-acquired pneumonia is only partly known. Up to this moment, many pathogens may have a role in establishing the infection but $S$. pneumoniae, $H$. influenzae, RSV and M. pneumoniae are responsible for more than half of the cases. Non-type b H. influenzae is an important cause of pneumonia, as are numerous serotypes of pneumococcus. The immunogenicity of the conjugate vaccines for $S$. pneumoniae and $H$. influenzae is typespecific. It can be calculated that by widespread use 
of currently available pneumococcal and $H$. influenzae type b conjugate vaccines, $30 \%$ of pediatric pneumonia cases could be prevented, depending on the serotype distribution of each of those pathogens in the region where vaccines are to be used. From the foregoing data, it is clear that, in addition to pneumococcal and Hib conjugate vaccines, an efficacious RSV vaccine is highly desirable, as well as non-type $\mathrm{b} H$. influenzae and $M$. pneumoniae vaccines, in order to reduce the pneumonia burden by at least $50 \%$ of present levels. This would be an important advance in medical care, particularly in regions of the world where childhood pneumonia is so common.

\section{References}

1. McCracken Jr G. Etiology and treatment of pneumonia. Pediatr Infect Dis J 2000; 19:373-7.

2. Murphy T.F., Henderson F.W., Clyde W.A., et al. Pneumonia: an eleven-year study in a pediatric practice. Am J Epidemiol 1981; 113:12-21.

3. Jokinen C., Heiskanen L., Juvonen H., et al. Incidence of community-acquired pneumonia in the population of four municipalities in eastern Finland. Am J Epidemiol 1993; $137: 977-88$.

4. Pechere J.C. Community-acquired pneumonia in children. Worthing, UK: Cambridge Medical Publications; 1995.

5. Denny F.W., Loda F.A. Acute respiratory infections are the leading cause of death in children in developing countries. Am J Trop Med Hyg 1986;35:1-2.

6. PAHO/WHO. ARI in the Americas - Biannual Reports No. 1, December 1995. PAHO/HCP/HCT/ARI/95.27.

7. Benguigui Y. Infecções Respiratórias Agudas: Fundamentos Técnicos das Estratégias de Controle. Washington, D. C.: OPS; c1997.

8. Geddes A.M. Empiric therapy in lower respiratory tract infection: an ongoing challenge. J Chemother 1997;9(Suppl 3):5-9.

9. Juvén T., Mertsola J., Waris M., et al. Etiology of community-acquired pneumonia in 254 hospitalized children. Pediatr Infect Dis J 2000; 19:293-8.

10. Nohynek H., Eskola J., Laine E., et al. The causes of hospital-treated lower respiratory tract infection in children. Am J Dis Child 1991;145:618-22.

11. Wall R.A., Corrah P.T., Mabey D.C.W., Greenwood B.M.. The etiology of lobar pneumonia in the Gambia. Bull WHO 1986;64:553-8.

12. Silverman M., Stratton D., Diallo A., et al. Diagnosis of acute bacterial pneumonia in Nigerian children. Arch Dis Child 1977;52:925-31.
13. Ramsey B.W., Marcuse E.K., Foy H.M., et al. Use of bacterial antigen detection in the diagnosis of pediatric lower respiratory tract infections. Pediatrics 1986;78:1-9.

14. Claesson B.A., Trollfors B., Brolin I., et al. Etiology of community-acquired pneumonia in children based on antibody responses to bacterial and viral antigens. Pediatr Infect Dis J 1989;8:856-62.

15. Barker J., Gratten M., Riley I., et al. Pneumonia in children in the Eastern Highlands of Papua New Guinea: a bacteriologic study of patients selected by standard clinical criteria. J Infect Dis 1989;159:348-52.

16. Mulholland K. Magnitude of the problem of childhood pneumonia. Lancet 1999;354:590-2.

17. Forgie I.M., O’Neill K.P., Lloyd-Evans N., et al. Etiology of acute lower respiratory tract infections in Gambian children: I. Acute lower respiratory tract infections in infants presenting at the hospital. Pediatr Infect Dis J 1991;10:33-41.

18. Ruuskanen O., Nohynek H., Ziegler T., et al. Pneumonia in childhood: etiology and response to antimicrobial therapy. Eur J Clin Microbiol Infect Dis 1992; 11:217-23.

19. Korppi M., Leinonen M. Pneumococcal immune complexes in the diagnosis of lower respiratory infections in children. Pediatr Infect Dis J 1998; 17:992-5.

20. Adegbola R.A., Obaro S.K. Diagnosis of childhood pneumonia in the Tropics. Ann Trop Med Parasitol 2000;94:197-207.

21. Schuster A., Pino M. Neira, et al. La puncion biopsia pulmonar como metodo diagnóstico de las neumopatias de la infancia. Pediatria (Chile) 1966;9:9-12.

22. Rozov T., Sakane P.T., Novaes J.R.C., et al, Contribuição ao estudo da etiologia das pneumopatias da infância, por meio da aspiração pulmonar transcutânea. Pediatr Prat (São Paulo) 1974;45:221-32.

23. Ferreira O.S., Magalhães M., Veras A. Bacteriologia das pneumonias da criança, em material colhido por punção pulmonar, na cidade do Recife. J Pediatr (Rio de Janeiro) 1978;44:160-3.

24. Riley I., Carrad E., Gratten H., et al. The status of research on acute respiratory infections in children in Papua New Guinea. Pediatr Res 1983; 17:1041-3.

25. Shann F., Gratten M., Germer S., et al. Aetiology of pneumonia in children in Goroka Hospital, Papua New Guinea. Lancet 1984;2:537-41.

26. Ikeogu M.O. Acute pneumonia in Zimbabwe: bacterial isolates by lung aspiration. Arch Dis Child 1988;63:1266-7.

27. Bullowa J.G.M. The reliability of sputum typing and its relation to serum therapy. JAMA 1935;105:1512-23.

28. Shann F. Etiology of severe pneumonia in children in developing countries. Pediatr Infect Dis J 1986;5:247-52. 
29. Turner R.B., Lande A.E., Chase P., et al. Pneumonia in pediatric outpatients: cause and clinical manifestations. J Pediatr 1987; 111:194-200.

30. Ausina V., Coll P., Sambeat M., et al. Prospective study on the etiology of community-acquired pneumonia in children and adults in Spain. Eur J Clin Microbiol Dis 1988; $7: 342-7$.

31. Heiskanen-Kosma T., Korppi M., Jokinen C., et al. Etiology of childhood pneumonia: serological results of a prospective population-based study. Pediatr Infect Dis J 1998; 17:986-91.

32. Wubbel L., Muniz L., Ahemed A., et al. Etiology and treatment of community-acquired pneumonia in ambulatory children. Pediatr Infect Dis J 1999; 18:98-104.

33. Nichol K.P, Cherry J.D. Bacterial-viral interrelations in respiratory infections of children. N Engl J Med 1967;277:667-72.

34. Simoes E.A. Respiratory syncytial virus infection. Lancet 1999;354(9181):847-52.

35. Ghafoor A., Nomani N.K., Ishaq Z., et al. Diagnoses of Acute Lower Respiratory Tract Infections in Children in Rawalpindi and Islamabad, Pakistan. Rev Infect Dis 1990;12(Suppl 8):S907-14.

36. Tupasi T.E., Lucero M.G., Magdangal D.M., et al. Etiology of Acute Lower Respiratory Tract Infections in Children from Alabang, Metro Manila. Rev Infect Dis 1990;12(S8):S929-S39.

37. Vardas E., Blaauw D., McAnerney J. The epidemiology of respiratory syncytial virus (RSV) infections in South African children. S Afr Med J 1999;89:1079-84.

38. Chan P.K., Sung R.Y., Fung K.S., et al. Epidemiology of respiratory syncytial virus infection among paediatric patients in Hong Kong: seasonality and disease impact. Epidemiol Infect 1999; 123:257-62.

39. Carballal G., Videla C., Sequeira M.D., et al. Respiratory syncytial virus: changes in prevalence of subgroups A and B among Argentinian children, 1990-1996. J Med Virol 2000;61:275-9.

40. Muhe L., Tilahun M., Lulseged S., et al. Etiology of pneumonia, sepsis and meningitis in infants younger than three months of age in Ethiopia. Pediatr Infect Dis J 1999;18(10Suppl):S56-61.

41. Sonoda S., Gotoh Y., Bann F., Nakayama T. Acute lower respiratory infections in hospitalized children over a 6 year period in Tokyo. Pediatr Int 1999;41:519-24.

42. Miranda-Novales G., Solorzano-Santos F., LeanosMiranda B., et al. Blood culture and respiratory syncytial virus identification in acute lower respiratory tract infection. Indian J Pediatr 1999;66:831-6.

43. Hussey G.D., Apolles P., Arendse Z., et al. Respiratory syncytial virus infection in children hospitalised with acute lower respiratory infection. S Afr Med J 2000;90:509-12.
44. Schwarze J., Hamelmann E., Bradley K.L., et al. Respiratory syncytial virus infection results in airway hyperresponsiveness and enhanced airway sensitization to allergen. J Clin Invest 1997;100:226-33.

45. Chaudhry R., Nazima N., Dhawan B., Kabra S.K. Prevalence of Mycoplasma pneumoniae and Chlamydia pneumoniae in children with community acquired pneumonia. Indian J Pediatr 1998;65:717-21.

46. Ouchi K., Komura H., Fujii M., et al. Chlamydia pneumoniae infection and Mycoplasma pneumoniae infection in pediatric patients. Kansenshogaku Zasshi 1999;73:1177-82.

47. Dudko S., Plusa T., Chcialowski A., et al. Serological screening examinations of atypical pathogens (Mycoplasma pneumoniae, Chlamydia pneumoniae) in respiratory tract infection. Pol Merkuriusz Lek 2000; 7:23-6.

48. Block S., Hedrick J., Hammerschlag M., et al. Mycoplasma pneumoniae and Chlamydia pneumoniae in pediatric community-acquired pneumonia: comparative efficacy and safety of clarithromycin versus Erythromycin ethylsuccinate. Pediatr Infect Dis J 1995; 14:471-7.

49. Harris J.A., Kolokathis A., Campell M., et al. Safety and efficacy of azithromycin in the treatment of communityacquired pneumonia in children. Pediatr Infect Dis J 1998; $17: 865-71$.

50. Brunell P.A., Bass J.W., Daum R.S., et al. American Academy of Pediatrics. Committee on Infectious Diseases. Recommendations for using pneumococcal vaccine in children. Pediatrics 1985;75:1153-8.

51. Black S., Shinefield H., Fireman B., et al. Efficacy, safety and immunogenicity of heptavalent pneumococcal conjugate vaccine in children. Pediatr Infect Dis J 2000; 19:187-95.

52. Overturf G.D. American Academy of Pediatrics. Committee on Infectious Diseases. Technical report: prevention of pneumococcal infections, including the use of pneumococcal conjugate and polysaccharide vaccines and antibiotic prophylaxis Pediatrics 2000;106:367-76.

53. Di Fabio J.L., Homma A., Quadros C. Pan American Health Organization Epidemiological Surveillance Network for Streptococcus pneumoniae. Microbial Drug Resistance 1997;3:131-3.

54. Eskola J., Anttila M. Pneumococcal conjugate vaccines. Pediatr Infect Dis J 1999; 18:543-51.

55. De Cunto Brandileone M.C., Diaz Vieira V.S., Casagrande S.T., et al. Prevalence of serotypes and antimicrobial resistance of Streptococcus pneumoniae strains isolated from Brazilian children with invasive infections. Microbial Drug Resist 1997;3:141-6.

56. CDC. Progress toward elimination of Haemophilus influenzae type $\mathrm{b}$ among infants and children: United States, 1987-1993. MMWR 1994;43:144-8. 
57. Ward J.I., Zangwill K,M. Haemophilus influenzae. In: Feigin R.D., Cherry J.D. [eds]. Textbook of Pediatric Infectious Diseases. $4^{\text {th }}$ ed. Philadelphia: W.B. Saunders Company; 1998, p1464-1482.

58. Klein J.O. Bacterial Pneumonias. In: Feigin R.D., Cherry J.D. [eds]. Textbook of Pediatric Infectious Diseases. $4^{\text {th }}$ ed. Philadelphia: W.B. Saunders Company; 1998, p273-284.

59. Liston T.E., Foshee W.S. Invasive disease due to nontypable Haemophilus influenzae in children. South Med J 1982;75:753-54.

60. Malhotra A., Krilov L.R. Influenza and respiratory syncytial virus. Update on infection, management, and prevention. Pediatr Clin North Am 2000;47:353-72.

61. Kneyber M.C., Moll H.A.,de Groot R. Treatment and prevention of respiratory syncytial virus infection. Eur J Pediatr 2000;159:399-411.

62. Kahn J.S. Respiratory syncytial virus vaccine development. Curr Opin Pediatr 2000;12:257-62.

63. Peebles Jr R.S., Sheller J.R., Collins R.D., et al. Respiratory syncytial virus (RSV) induced airway hyperresponsiveness in allergically sensitized mice is inhibited by live RSV and exacerbated by formalininactivated RSV. J Infect Dis 2000; 182:671-7.

64. Crowcroft N.S., Cutts F., Zambon M.C. Respiratory syncytial virus: an underestimated cause of respiratory infection, with prospects for a vaccine. Commun Dis Public Health 1999;2:234-41.

65. Cimolai N., Cheong A.C., Morrison B.J., Taylor GP. Mycoplasma pneumoniae reinfection and vaccination: protective oral vaccination and harmful immunoreactivity after re-infection and parenteral immunization. Vaccine 1996; 14:1479-83. 